\title{
The Effect of Hospital and Surgeon Volume on Racial Differences in Recurrence-Free Survival After Radical Prostatectomy
}

\author{
Kyna M. Gooden, PhD ${ }^{\star}$, Daniel L. Howard, PhD*, William R. Carpenter, PhD ${ }^{\star} \ddagger$, April P. \\ Carson, PhD ${ }^{\star}$, Yhenneko J. Taylor, MS ${ }^{\star}$, Sharon Peacock, MPA $\S$, and Paul A. Godley, MD, \\ PhDt, \\ "The Institute for Health, Social, and Community Research, Shaw University, Raleigh, North \\ Carolina \\ †Lineberger Comprehensive Cancer Center, University of North Carolina at Chapel Hill, Chapel Hill, \\ North Carolina \\ ‡Department of Health Policy and Administration, School of Public Health, University of North \\ Carolina at Chapel Hill, Chapel Hill, North Carolina \\ $\S$ The Carolinas Center for Medical Excellence, Cary, North Carolina \\ IDivision of Hematology/Oncology, University of North Carolina at Chapel Hill, Chapel Hill, North \\ Carolina
}

\begin{abstract}
Objective-This study investigates associations between hospital and surgeon volume, and racial differences in recurrence after surgery for prostate cancer.

Methods-Data from the 1991 to 2002 Surveillance, Epidemiology, and End-Results-Medicare database were examined for 962 black and 7387 white men who received surgery for prostate cancer within 6 months of diagnosis during 1993-1999. Cox regression models were used to estimate the relationships between volume (grouped in tertiles), recurrence or death, and race, controlling for age, Gleason grade, and comorbidity score.
\end{abstract}

\begin{abstract}
Results-Prostate cancer recurrence-free survival rates improved with hospital and surgical volume. Black men were more likely to experience recurrence than white men [hazard ratio $(\mathrm{HR})=$ $1.34 ; 95 \%$ confidence interval (CI): $1.20-1.50]$. Stratification by hospital volume revealed that racial differences persisted for medium and high volume hospitals, even after covariate adjustments (medium HR $=1.30,95 \%$ CI: $1.04-1.61$; high HR $=1.36,95 \%$ CI: 1.07-1.73). Racial differences persisted within medium and high levels of surgeon volume as well (medium $\mathrm{HR}=1.43,95 \% \mathrm{CI}$ : $1.10-1.85$; high $\mathrm{HR}=1.57,95 \%$ CI: $1.14-2.16$ ).
\end{abstract}

Conclusions-High hospital and physician volumes were not associated with reduced racial differences in recurrence-free survival after prostate cancer surgery, contrary to expectation. This study suggests that social and behavioral characteristics, and some aspects of access, may play a larger role than organizational or systemic characteristics with regard to recurrence-free survival for this population.

\footnotetext{
(C) 2008 by Lippincott Williams \& Wilkins

Reprints: Daniel L. Howard, PhD, The Institute for Health, Social and Community Research, Shaw University, 118 E. South Street, Raleigh, NC 27601. howardd@shawu.edu.
} 


\section{Keywords}

prostate cancer; survival; hospital volume; surgeon volume; racial disparities

Prostate cancer is the leading cancer diagnosis among American men and the second most common cause of cancer-related death among this group. An estimated 218,890 new cases of prostate cancer will be diagnosed in the United States in 2007, with an estimated 27,050 deaths. ${ }^{1}$ Prostate cancer incidence and mortality rates for both blacks and whites have declined since 1991-1992; however, the incidence and mortality rates for blacks remain approximately twice as high as those for whites. ${ }^{2,3}$

Treatment options for prostate cancer vary by stage of disease, patient preference, and other comorbidities. Studies have shown treatment ${ }^{4,5}$ and treatment outcomes ${ }^{6,7}$ to differ by race. Black men are less likely to get aggressive definitive treatment compared with whites. ${ }^{4,5}$ Although survival after treatment for localized prostate cancer has been shown to be lower for blacks compared with whites, particularly after surgery, ${ }^{6}$ research to identify treatment-specific variables related to racial differences in treatment outcomes is lacking.

To this end, there is a growing interest in understanding how physician and hospital characteristics affect patient care and outcomes. Hospital volume, or the number of procedures performed, is associated with more favorable outcomes for many surgical procedures, including pancreaticoduodenectomy, ${ }^{8,9}$ esophagectomy, 9 prostatectomy, 10 and liver resection. 9 Previous reviews found that the majority of studies support a positive relationship between volume and outcome of initial cancer treatment for both physician and hospital. ${ }^{11}$

Other characteristics have emerged as factors related to patient outcomes. In general, studies show that physicians of black patients tend to be less well trained (eg, board certification), and have less access to important clinical resources (eg, high-quality diagnostic imaging) than physicians of white patients. ${ }^{12}$ Location, size, and type of hospital have also been associated with treatment outcomes and quality of care. $13^{-} 15$ These concepts and research findings suggest that, if blacks are more likely to receive care at hospitals and by physicians that differ in experience from the hospitals and physicians that whites use, this could account for some of the racial differences that are seen in treatment outcomes after prostatectomy.

Previous research by Cohen and associates used the Surveillance, Epidemiology, and EndResults (SEER)-Medicare database to examine patients diagnosed with clinically localized prostate cancer from 1988 to 1996, and found racial disparities in both prostate cancer specific and all-cause recurrence-free survival, with the greatest racial difference in survival found in patients after prostatectomy. ${ }^{16}$ However, the study did not analyze whether this disparity is related to the experience level (or volume) of hospitals and surgeons who perform the prostate surgeries. This study expands upon the work of Cohen and associates to examine the relationship between physician/hospital volume and the racial disparity in survival after surgery for prostate cancer.

\section{METHODS}

\section{Study Population}

Data are from the 1991 to 2002 SEER-Medicare database, described in detail elsewhere. ${ }^{17}$

Briefly, SEER-Medicare is a collaborative effort between the SEER program and the Medicare program, offering a comprehensive dataset of cancer diagnosis and treatments as well as other diseases and conditions that may influence treatment and survival. The SEER program is a cancer registry of the National Cancer Institute, collecting data from various areas of the United States on cancer diagnoses, histology, grade, stage, and first treatment, as well as patient 
demographic characteristics, follow-up of vital status, and cause of death (if applicable). The Medicare program provides health insurance coverage to approximately 97\% of Americans 65 years of age or older. Medicare data provides health services claims for care provided by physicians, hospitals, home health care agencies, nursing facilities, and hospice programs.

For this study, patients aged 65 years or older diagnosed with prostate cancer from 7 SEER regions (Atlanta, Connecticut, Detroit, San Francisco, Seattle, San Jose, and Los Angeles) were included. These regions were chosen because they included larger percentages of blacks. Patients were evaluated for treatment after diagnosis. Those who underwent surgery with curative intent for prostate cancer during 1993-1999 and within 6 months of diagnosis were included in the analytic dataset. Surgery was identified using the Healthcare Common Procedure Coding System/Current Procedural Terminology (HCPCS/CPT), International Classification of Diseases, ninth revision (ICD-9), and SEER site-specific surgery codes. Surgery with curative intent was defined by codes for prostatectomy (HCPCS/CPT codes 55810, 55812, 55815, 55821, 55831, 55840, 55842, 55845, 55866; ICD-9 codes 60.3, 60.4, 60.5, 60.6; SEER site-specific codes 30-90) and lymph node procedures (ICD-9 codes 40.1, $40.2,40.3,40.5)$, because the performance of lymph node procedures commonly indicates a planned prostatectomy that was aborted due to the cancer's advanced stage, which was belatedly diagnosed through detection of disease in the lymph nodes during the procedure. Patients were excluded from this study if they lacked continuous Medicare coverage, were enrolled in an HMO, or had an ambiguous date of death.

\section{Measurement of Variables}

The main predictor variables, hospital and physician volume, were obtained from the Medicare claims data. Volume is defined as the number of prostate surgeries (identified through prostatectomy and lymph node procedure codes) performed during the 8 quarters before the patient's surgery date. Volume was calculated for the hospital and performing physician of each study patient individually at the time of the patient's surgery. Subjects were included if they had a prostatectomy, but for several, the performing physician or hospital was missing from the record listing the procedure, and so volume for these physicians and hospitals was not calculable. Hospital and physician volume were categorized into tertiles, corresponding to low (hospital: 0-63 procedures; physician: 0-9 procedures), medium (hospital: 64-116 procedures; physician: 10-19 procedures), and high (hospital: 117-371 procedures; physician: 20-77 procedures).

The Medicare claims data were also used to derive a comorbidity index score designed specifically for SEER-Medicare. ${ }^{18}$ Race (black, white), age (65-69, 70-74, 75+), and Gleason grade (well differentiated, moderately differentiated, poorly differentiated) were obtained from the SEER data. Cancer stage was obtained from SEER data, which provides prostate cancer staging based on designations by the American Joint Committee on Cancer (AJCC) (stages 14) as well as a simplified SEER historic stage (localized/regional and distant). We used the AJCC stage for classifying patients in our study. Where AJCC stage was missing, SEER historic stage was used. ${ }^{19}, 20$ Patients were classified as unstaged if insufficient information was available for staging.

Consistent with previous examinations of prostate cancer treatment outcomes, $10,16^{, 21}$ our endpoints were death or prostate cancer recurrence, captured by any subsequent treatment for prostate cancer at least 6 months or more after surgery, as found in the Medicare claims data. This window is chosen because it allows adequate time to initiate treatment, even after the common multidisciplinary consultations that occur after diagnosis. Treatment for prostate cancer includes radiation (HCPCS/CPT codes 77261-77799 excluding 77600-77620, ICD-9 codes 92.2, V58.0, V66.1, V67.1; revenue center codes 0330, 0333), orchiectomy (HCPS/CPT codes 54520, 54522, 54530, 54535, 54690; ICD-9 codes 62.3, 62.41, 62.42), and hormonal 
therapy (HCPS/CPT codes J1050, J1051, J1950, J3315, J9202, J9217, J9218, J9219, J9165). To account for adjuvant therapy, hormonal therapy was only considered as treatment for prostate cancer recurrence if claims were found 6 months or more after surgery, and no claims were found in the window 30 days before diagnosis to 180 days after diagnosis. In addition, if a hormonal therapy claim fell within this window, a 1-year interval between this claim and subsequent claims was necessary in order for hormonal therapy to be categorized as a recurrence event. All-cause mortality was recorded as indicated by the vital status variable in Medicare claims data and is comprehensive of prostate-specific mortality and causes of death that may have resulted from health conditions exacerbated by prostate cancer or prostatectomy. If the Medicare vital status variable was missing, the SEER death indicator was used $(n=13)$. Recurrence-free survival was measured in months from date of prostate surgery to the first event, recurrence or death.

\section{Statistical Analysis}

Descriptive analyses were performed to assess baseline characteristics by race. Unadjusted recurrence-free survival rates by race and volume were estimated using Kaplan-Meier survival curves and compared using Log-rank statistics. Because median survival was not reached in this sample (more than $50 \%$ of the sample remained alive), we focus on the 25th percentile of recurrence-free survival time, which is the time at which recurrence-free survival probability is 0.75. Hazard ratios from Cox regression models were examined separately for each level of hospital and physician volume8 to investigate whether racial differences in recurrence-free survival persisted within different categories of volume. To assess potential bias due to our selection of volume cut points we conducted sensitivity analyses using multiple functional forms of volume, including as binary variables (quartiles), and continuous measures, both as a simple linear function and with more complex functions (quadratic and cubic splines). Results showed no change in direction or magnitude of HR estimates for the race effect. Additionally, physician volume did not reach statistical significance in any models simultaneously adjusting for both hospital and physician volume. Models were assessed for confounding by age, cancer stage, Gleason grade, comorbidity score, bed count, and teaching hospital status. Confounding was based on a $10 \%$ or greater change in the magnitude of the main effect when the potential confounder was included in the model.22 The final models were adjusted for age, Gleason grade, comorbidity score, cancer stage, and SEER site. Statistical tests were adjusted for clustering, correcting for within-hospital correlations. All analyses were conducted using Statistical Analysis System (SAS), Version 9 (SAS Institute, Cary, NC).

\section{RESULTS}

A total of 8349 patients ( 962 black, 7387 white) were included in this analysis. The mean age was 69.3 ( $\mathrm{SD} \pm 3.7$ ) for blacks and 69.6 ( $\mathrm{SD} \pm 3.7$ ) for whites. Blacks were more likely than whites to have poorly differentiated tumors $(26.7 \%$ vs. $21.5 \%, P<0.001)$ and a stage IV cancer ( $8.8 \%$ vs. $7.3 \%, P<0.001$ ), though whites were more likely than blacks to have stage III cancer (32.5\% vs. $23.5, P<0.001)$. Blacks had greater comorbidity compared with whites as indicated both by a greater proportion with comorbidity scores of 2 or more $(9.0 \%$ vs. $3.5 \%, P<0.001)$ and a lower proportion with no comorbidity $(64.0 \%$ vs. $79.1 \%, P<0.001)$. Blacks were more likely to have surgeries at high volume hospitals $(40.7 \%$ vs. $30.4 \%, P<0.001$, overall; $43.9 \%$ vs. $32.3 \%, P<0.001$, among those with known volume) at NCI-designated Cancer Centers ( $16.2 \%$ vs. $8.2 \%, P<0.001$ ), and by lower volume surgeons (low volume: $31.5 \%$ vs. $25.9 \%$, $P<0.001$, overall; $50.3 \%$ vs. $34.0 \%, P<0.001$, among those with known volume; high volume: $11.4 \%$ vs. $25.3 \%, P<0.001$, overall; $18.3 \%$ vs. $33.3 \%, P<0.001$, among those with known volume) (Table 1). Black men were more likely than white men to receive surgery from a lowvolume physician regardless of the surgical volume of the hospital. 
Overall, patients in low volume hospitals experienced shorter recurrence-free survival times than patients in high volume hospitals (25th percentile: 45 months, 95\% CI: 42.0- 50.0 vs. 64 months, 95\% CI: 57.0-70.0). Likewise, patients with low or medium volume surgeons experienced similar recurrence-free survival times (25th percentile: 60 months, 95\% CI: 54.068.0 and 61 months, 95\% CI: 54.0-67.0), and both were lower than patients with high volume surgeons (25th percentile: 73 months, 95\% CI: 66.0-81.0).

Blacks experienced shorter recurrence-free survival times than whites. The 25 th percentile recurrence-free survival time was 43 months (95\% CI: 36.0-47.0) for blacks and 55 months (95\% CI: 52.0 -58.0) for whites. As presented in Figure 1 and Figure 2, stratification by volume presents similar results, with considerable racial disparities in recurrence-free survival that persist among high-volume and low-volume hospitals and physicians, most notably among high-volume hospitals and physicians. Among high-volume hospitals, the 25 th percentile recurrence-free survival time was 45 months (95\% CI: $37.0-55.0)$ for blacks, and 68 months (95\% CI: 62.0 -76.0) for whites. Similarly among high-volume physicians, the 25 th percentile recurrence-free survival was 43 months for blacks and 77 months (95\% CI: 68.0-83.0) for whites.

Multivariate analyses reveal black race as a significant predictor of recurrence or death among high- and medium-volume hospitals, even after adjusting for age, Gleason grade, comorbidity score, cancer stage, and SEER site (high-volume adjusted HR = 1.36, 95\% CI: 1.07-1.73; medium-volume adjusted HR $=1.30,95 \% \mathrm{CI}$ : $1.04-1.61$ ) (Table 2). When comparing physician volumes, black race remained a significant predictor of recurrence or death for patients treated by high- and medium-volume physicians as well (high-volume adjusted HR = 1.57, 95\% CI: $1.14-2.16$; medium-volume adjusted HR $=1.43$, 95\% CI: $1.10-1.85$ ) (Table 3). Racial differences among low-volume hospitals or physicians did not reach statistical significance.

\section{DISCUSSION}

The present study sought to build upon previous research by examining hospital and physician volume as a possible explanatory variable for the disparities in outcomes after prostate surgery. We hypothesized that a portion of the racial differences in prostate cancer treatment outcomes may be explained by different probabilities of seeking care from hospitals and physicians with differing levels of experience: white men may experience better outcomes due to a greater utilization of high-volume hospitals and physicians. This hypothesis was not confirmed-the poorer outcomes experienced by black men were not explained by their differential utilization of less experienced hospitals and/or surgeons. Instead, the racial disparity in outcomes persisted regardless of hospital or physician volume.

The study results suggest that black patients were more likely to experience recurrence or death than were white patients, even when controlling for surgical volume, and confirms previous findings that demonstrate a link between prostate cancer surgical volumes and outcomes: both high physician volume and high hospital volume were associated with better outcomes. ${ }^{10}$ They also demonstrate previously-observed racial differences in both recurrence ${ }^{16}$ and mortality ${ }^{6}$ after surgery for prostate cancer.

Had our hypothesis been confirmed, as demonstrated through high-volume facilities and surgeons being associated with attenuation in racial differences in outcomes, our findings may have suggested an opportunity for a policy-level intervention to address racial disparities. For example, health plans - including Federal payers - could have been encouraged to improve the outcomes of their minority beneficiaries by taking a proactive role in directing them to high-volume providers. Health systems and their medical staffs would be encouraged to look 
internally at the hospitals and surgeons who typically serve their communities' higher-risk populations and consider a consolidation of services to improve prostate surgery experience among, perhaps, a more limited number of their constituent hospitals and physicians. Other policy changes could encourage more experienced surgeons to consult on prostate cancer surgeries to mentor less experienced surgeons, or, similarly, encourage ongoing health education partnerships between higher and lower volume hospitals to extend the benefits of such mentoring and training to their surgeons and staff to improve patient outcomes. Although such policies may improve overall outcomes for all populations, our research shows that such interventions may be of limited utility in addressing the differences in outcomes between blacks and whites.

The decision to have surgery is a function of many known factors, including the stage of cancer, doctor recommendations, and patient preferences. Racial differences that transcend these characteristics and persist despite the experience of the provider may point to additional characteristics, such as tumor heterogeneity. Approximately $32 \%$ of black patients and $40 \%$ of white patients were classified as stage III or IV postsurgery, suggesting that white men with advanced disease were more likely than black men to receive surgery. If this were the case, one would expect white patients in this population to have less favorable outcomes in terms of recurrence, and possibly mortality. However, it is possible that surgeons are more likely to be aggressive with white patients with high-risk localized prostate cancer, which post-surgery is restaged as stage III (and up-coded in the registry data). Black patients with high risk may be guided toward, or inclined to seek, another treatment such as radiation therapy, which would not have subsequent pathologic staging (or up-coding in the registry). In addition, even within each tumor stage, tumors can vary widely with regard to tumor aggressiveness. Descriptive statistics show that blacks were more likely than whites to have poorly differentiated tumors. Thus, the blacks in our study may have had more aggressive disease within these stages.

Our study has several limitations. Volume is a proxy for experience and we examined the 8 quarters before surgery to determine volume levels. Although black men were more likely to have missing data on physician volume than were white men, additional analysis revealed no other discernible patterns in the data to suggest either its cause, the presence of bias, or any direction of bias. Also regarding volume, our data were unable to capture the absolute volume of surgeons, which would include any surgeries performed on patients who did not participate in Medicare (eg, younger than 65 years), or on individuals first diagnosed outside the SEER regions included in our study. A surgeon may also be very experienced in prostate cancer surgeries but was limited in the number performed during this study period due to a myriad of reasons. Thus, our definition of volume may be different from true experience; however, the risk and effect of bias resulting from age-limited analysis (eg, limiting to those age 65 and older) are likely to be limited when considering that previous research has found that the rank order of the hospitals by volume of surgery on patients aged 65 and older to be very similar to the rank order of the hospitals when including the absolute numbers of cancer surgeries performed (eg, all ages).$^{9}$ Overall, research consistently demonstrates volume as a predictor of patient outcomes after major procedures, $8^{-} 11$ warranting its merit as a proxy for experience.

Our study used claims data to measure recurrence-free survival using a method that has been validated ${ }^{23}$ and used in multiple previous studies of prostate cancer. ${ }^{16,21}$ Using this method, our measures of recurrence-free survival are likely conservative compared with true prostate cancer recurrence as this method is not able to capture recurrent disease treated with selfadministered prescription drugs or those that required no treatment, such as the "watch and wait" strategy; however, the probability of these scenarios is small and, therefore, so is any likely impact on our findings. 
Our decision to use tertiles as opposed to quartiles as cut-points to categorize volume preserved statistical power and precision; however, it may have done so at the risk of losing some insight into the nuances of the volume-outcome relationship. The direction of any potential bias is unclear, and extensive sensitivity analyses of the functional form of the volume measure suggest that use of tertiles was appropriate, and results using other functional forms would not alter our conclusions.

Bivariate analysis indicated that black men were more likely to see low-volume physicians, regardless of the hospital volume. It would have been interesting and may have yielded additional explanatory insight to examine the interaction of physician volume and hospital volume in the analytic model, particularly with regard to the effect of seeing low-volume physicians at high-volume hospitals, as experienced disproportionately by blacks in our study. However, study sample size precluded such examination in the analytic model. Regardless, the tendency of black men to see low-volume physicians in all settings warrants further investigation with a sufficiently large sample size to examine this question more closely.

In addition to prostate cancer-specific recurrence-free survival, overall recurrence-free survival was chosen as an end point and not prostate-specific mortality, because both hospital and physician experience with prostatectomy can influence clinical outcomes attributable to noncancer causes, such as anesthesia, perioperative mortality, and surgery-related sequelae, and this end point allows us to capture these deaths as well as mortality related directly to prostate cancer. As also found in other cancer outcomes studies, our previous research found trends and racial disparities in all-cause mortality to closely mirror prostate-cancer specific mortality, ${ }^{6,16}$ and we have no reason to believe there to be a difference or any consequent bias in this analysis, which is based on the same SEER-Medicare population.

In conclusion, racial differences in recurrence or mortality after surgery for prostate cancer did not consistently decrease with increasing hospital or physician volume as expected. Patterns of care for black men differed from white men in that they tended to undergo surgery at highvolume hospitals, and they tended to be operated on by low-volume surgeons. Thus, the poorer outcomes experienced by black men are not explained by less experienced hospitals or surgeons in these data, and so organizational or systemic interventions to address disparities through volume are not supported, while differences in basic biologic characteristics (eg, tumor aggressiveness), and social and behavioral characteristics remain likely origins of the demonstrated racial differences and points of intervention. Accordingly, future research should investigate other factors that could affect the racial disparity in recurrence and survival after prostate cancer surgery, such as lifestyle factors and clinical characteristics of the prostate tumor.

\section{Acknowledgments}

This study used the linked SEER-Medicare database. The interpretation and reporting of these data are the sole responsibility of the authors. The authors acknowledge the efforts of the Applied Research Program, NCI; the Office of Research, Development and Information, CMS; Information Management Services (IMS), Inc.; and the Surveillance, Epidemiology, and End Results (SEER) Program tumor registries in the creation of the SEER-Medicare database. The authors also acknowledge and thank Anna P. Schenck, PhD, from the Carolinas Center for Medical Excellence, Cary, NC for lending her expertise to this project.

Supported by the DoD CDMRP contract W81XWH-05-1-0208 (grant PC00907). Shaw investigators are also funded, in part, by National Institutes of Health NCMHD grant P60 MD000239 and Department of Health and Human Services AHRQ grant R24 HS013353. 


\section{REFERENCES}

1. American Cancer Society. Cancer Facts and Figures 2007. Atlanta, GA: American Cancer Society; 2007.

2. Ries, LAG.; Eisner, MP.; Kosary, CL., et al. SEER Cancer Statistics Review, 1975-2002. National Cancer Institute: Bethesda, MD; 2005. Available at, based on November 2004 SEER data submission, posted to the SEER web sitehttp://seer.cancer.gov/csr/1975_2002/

3. Chu KC, Tarone RE, Freeman HP. Trends in prostate cancer mortality among black men and white men in the United States. Cancer 2003;97:1507-1516. [PubMed: 12627516]

4. Tewari A, Horninger W, Pelzer AE, et al. Factors contributing to the racial differences in prostate cancer mortality. BJU Int 2005;96:1247-1252. [PubMed: 16287439]

5. Underwood W, Jackson J, Wei JT, et al. Racial treatment trends in localized/regional prostate carcinoma: 1992-1999. Cancer 2005;103:538-545. [PubMed: 15612083]

6. Godley PA, Schenck AP, Amamoo MA, et al. Racial differences in mortality among Medicare recipients after treatment for localized prostate cancer. J Natl Cancer Inst 2003;95:1702-1710. [PubMed: 14625261]

7. Moul JW, Douglas TH, McCarthy WF, et al. Black race is an adverse prognostic factor for prostate cancer recurrence following radical prostatectomy in an equal access health care setting. J Urol 1996;155:1667-1673. [PubMed: 8627850]

8. Birkmeyer JD, Finlayson SR, Tosteson AN, et al. Effect of hospital volume on in-hospital mortality with pancreaticoduodenectomy. Surgery 1999;125:250-256. [PubMed: 10076608]

9. Begg CB, Cramer LD, Hoskins WJ, et al. Impact of hospital volume on operative mortality for major cancer surgery. JAMA 1998;280:1747-1751. [PubMed: 9842949]

10. Begg CB, Riedel ER, Bach PB, et al. Variations in morbidity after radical prostatectomy. N Engl J Med 2002;346:1138-1144. [PubMed: 11948274]

11. Hillner BE, Smith TJ, Desch CE. Hospital and physician volume or specialization ion and outcomes in cancer treatment: importance in quality of cancer care. J Clin Oncol 2000;18:2327-2340. [PubMed: 10829054]

12. Bach PB, Pham HH, Schrag D, et al. Primary care surgeons who treat blacks and whites. N Engl J Med 2004;351:575-584. [PubMed: 15295050]

13. Lutfiyya MN, Bhat DK, Gandhi SR, et al. A comparison of quality of care indicators in urban acute care hospitals and rural critical access hospitals in the United States. Int J Qual Health Care 2007;19:141-149. [PubMed: 17442745]

14. Young GJJ, Meterko MP, Desai KRP. Patient satisfaction with hospital care: effects of demographic and institutional characteristics. Med Care 2000;38:325-334. [PubMed: 10718357]

15. Kupersmith J. Quality of care in teaching hospitals: a literature review. Acad Med 2005;80:458-466. [PubMed: 15851459]

16. Cohen JH, Schoenbach VJ, Kaufman JS, et al. Racial differences in clinical progression among Medicare recipients after treatment for localized prostate cancer (United States). Cancer Causes Control 2006;17:803-811. [PubMed: 16783608]

17. Warren JL, Klabunde CN, Schrag D, et al. Overview of the SEER-Medicare data: content, research applications, and generalizability to the United States elderly population. Med Care 2002;40:3-18.

18. Klabunde CN, Potosky AL, Legler JM, et al. Development of a comorbidity index using physician claims data. J Clin Epidemiol 2000;53:1258-1267. [PubMed: 11146273]

19. Jones MP. Indicator and stratification methods for missing explanatory variables in multiple linear regression. J Am Stat Assoc 1996;91:222-223.

20. Shavers VL, Brown ML, Potosky AL, et al. Race/ethnicity and the receipt of watchful waiting for the initial management of prostate cancer. J Gen Intern Med 2004;19:146-155. [PubMed: 15009794]

21. Lu-Yao GL, Potosky AL, Albertsen PC, et al. Follow-up prostate cancer treatments after radical prostatectomy: a population-based study. J Natl Cancer Inst 1996;88:166-173. [PubMed: 8632490]

22. Rothman, K.; Greenland, S. Modern Epidemiology. Philadelphia, PA: Lippincott-Raven; 1998.

23. Earle CC, Nattinger AB, Potosky AL, et al. Identifying cancer relapse using SEER-Medicare data. Med Care 2002;40:IV-75-IV-81. 


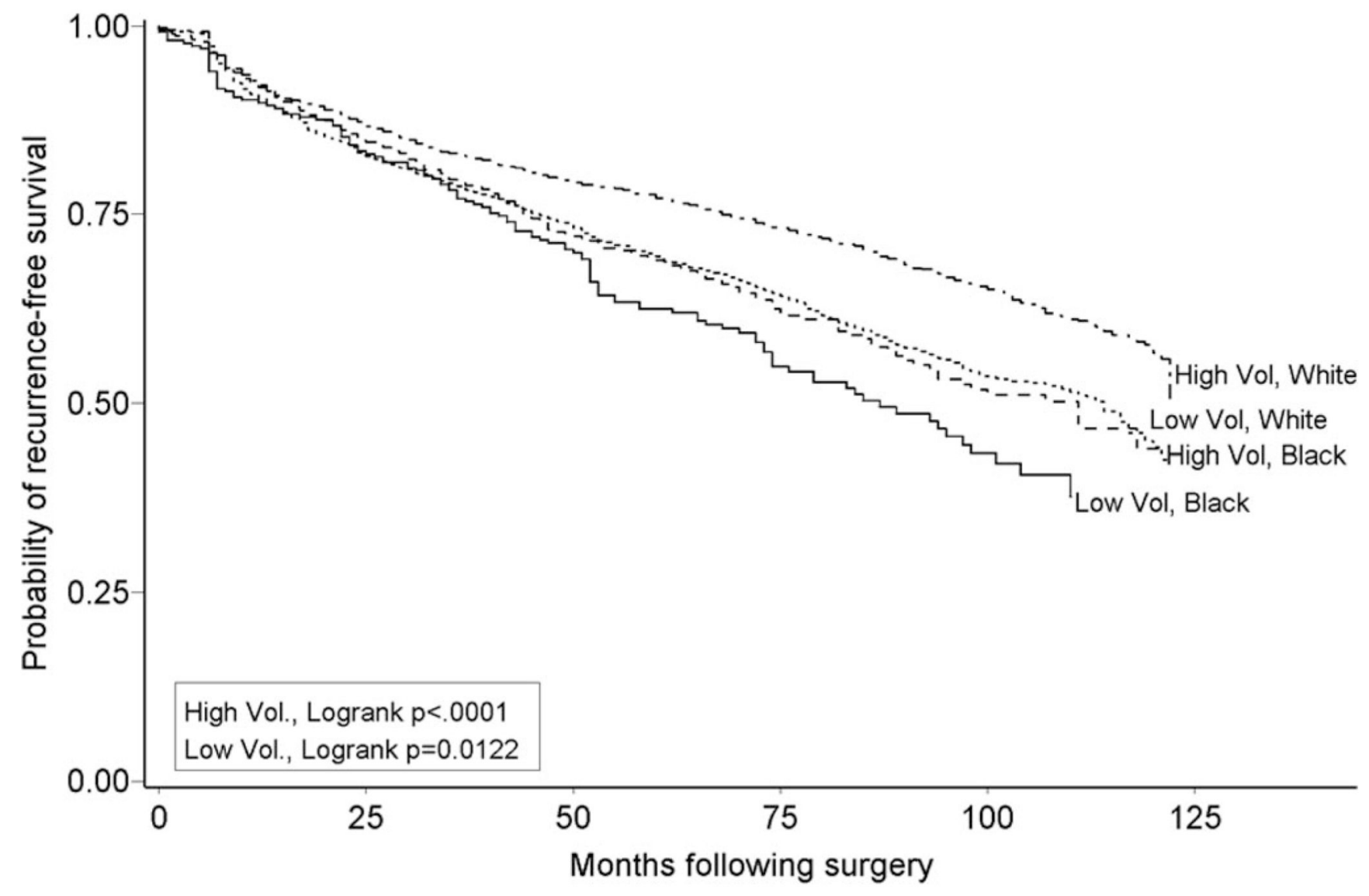

FIGURE 1.

Recurrence-free survival after prostate cancer surgery by hospital volume and race, SEERMedicare 1991-2002. 


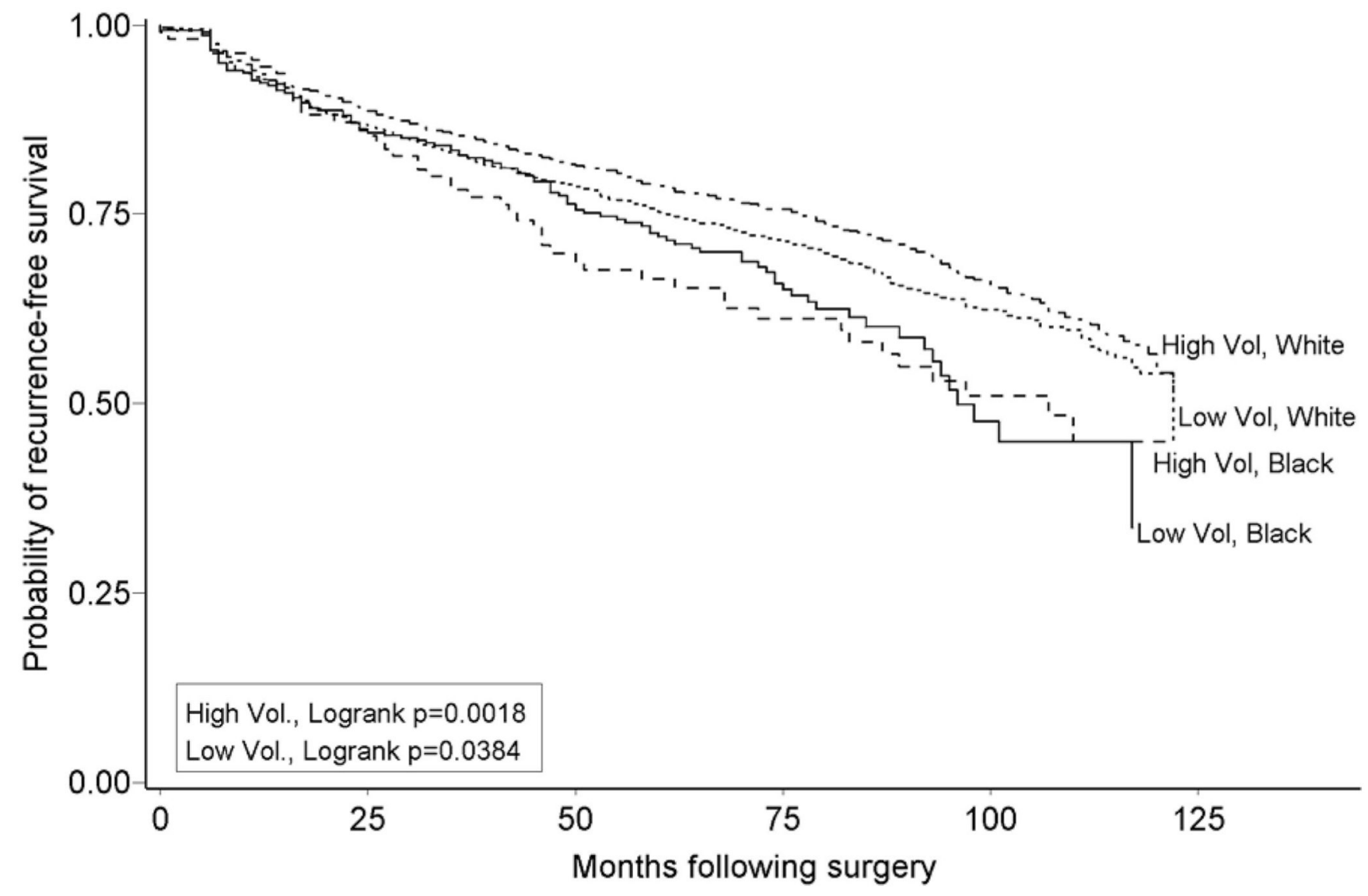

FIGURE 2.

Recurrence-free survival after prostate cancer surgery by physician volume and race, SEERMedicare 1991-2002. 
TABLE 1

Distribution of Characteristics by Patient Race, SEER-Medicare 1991-2002*

\begin{tabular}{|c|c|c|c|}
\hline & $\begin{array}{c}\text { Black } \\
\mathrm{N}=962\end{array}$ & $\begin{array}{c}\text { White } \\
\mathbf{N}=\mathbf{7 3 8 7}\end{array}$ & $P$ \\
\hline \multicolumn{4}{|l|}{ Patient characteristics } \\
\hline Age at diagnosis, mean (SD) & $69.3(3.7)$ & $69.6(3.7)$ & 0.006 \\
\hline Cancer stage $\mathrm{e}^{\dagger}, \mathrm{n}(\%)$ & & & $<0.001$ \\
\hline Stage 1 & $137(14.2)$ & $987(13.4)$ & \\
\hline Stage 2 & $156(16.2)$ & $1194(16.2)$ & \\
\hline Stage 3 & $226(23.5)$ & $2403(32.5)$ & \\
\hline Stage 4 & $85(8.8)$ & $539(7.3)$ & \\
\hline Localized/regional & $311(32.3)$ & $2002(27.1)$ & \\
\hline Unstaged & $47(4.9)$ & $262(3.5)$ & \\
\hline Gleason grade, n (\%) & & & $<0.001$ \\
\hline Well differentiated & $57(5.9)$ & $441(6.0)$ & \\
\hline Moderately differentiated & $607(63.1)$ & $5149(69.7)$ & \\
\hline Poorly differentiated & $257(26.7)$ & $1591(21.5)$ & \\
\hline Unknown & $41(4.3)$ & $206(2.8)$ & \\
\hline Comorbidity score, n (\%) & & & $<0.001$ \\
\hline 0 & $616(64.0)$ & $5843(79.1)$ & \\
\hline 1 & $209(21.7)$ & $1014(13.7)$ & \\
\hline $2+$ & $87(9.0)$ & $258(3.5)$ & \\
\hline Unknown & $50(5.2)$ & $272(3.7)$ & \\
\hline SEER site, n (\%) & & & $<0.001$ \\
\hline Atlanta & $188(19.5)$ & $665(9.0)$ & \\
\hline Connecticut & $76(7.9)$ & $962(13.0)$ & \\
\hline Detroit & $394(41.0)$ & $1551(21.0)$ & \\
\hline Los Angeles & $173(18.0)$ & $1647(22.3)$ & \\
\hline San Francisco & $88(9.1)$ & $710(9.6)$ & \\
\hline San Jose & $14(1.5)$ & $367(5.0)$ & \\
\hline Seattle-Puget Sound & $29(3.0)$ & $1485(20.1)$ & \\
\hline \multicolumn{4}{|l|}{ Hospital characteristics } \\
\hline Certified beds, mean (SD) & $564.3(238.0)$ & $401.1(237.5)$ & $<0.001$ \\
\hline NCI Cancer Center & & & $<0.001$ \\
\hline Yes & $156(16.2)$ & $608(8.2)$ & \\
\hline No & $736(76.5)$ & $6360(86.1)$ & \\
\hline Unknown & $70(7.3)$ & $419(5.7)$ & \\
\hline Physician volume, $\mathrm{n}(\%)^{\ddagger}$ & & & $<0.001$ \\
\hline Low: 0-9 & $303(50.3)$ & $1913(34.0)$ & \\
\hline Medium: 10-19 & $189(31.4)$ & $1836(32.7)$ & \\
\hline High: 20-77 & $110(18.3)$ & $1871(33.3)$ & \\
\hline Hospital volume, $\mathrm{n}(\%)^{\frac{\xi}{t}}$ & & & $<0.001$ \\
\hline
\end{tabular}




\begin{tabular}{|c|c|c|c|}
\hline & $\begin{array}{c}\text { Black } \\
\mathrm{N}=962\end{array}$ & $\begin{array}{l}\text { White } \\
\mathbf{N}=7387\end{array}$ & $\boldsymbol{P}$ \\
\hline Low: 0-63 & $266(29.8)$ & $2344(33.6)$ & \\
\hline Medium: 64-116 & $234(26.2)$ & $2375(34.1)$ & \\
\hline High: $138-371$ & $392(43.9)$ & $2249(32.3)$ & \\
\hline \multicolumn{4}{|l|}{ Hospital and physician volume $\$$} \\
\hline $\begin{array}{l}\text { Hospitals with low volume, } \mathrm{n} \\
(\%)\end{array}$ & & & 0.661 \\
\hline \multicolumn{4}{|l|}{ Physician volume } \\
\hline Low & $81(53.6)$ & $825(50.6)$ & \\
\hline Medium & $51(33.8)$ & $562(34.4)$ & \\
\hline High & $19(12.6)$ & $245(15.0)$ & \\
\hline Hospitals with medium volume, $\mathrm{n}(\%)$ & & & $<0.001$ \\
\hline \multicolumn{4}{|l|}{ Physician volume } \\
\hline Low & $60(41.4)$ & $488(25.2)$ & \\
\hline Medium & $53(36.6)$ & $727(37.5)$ & \\
\hline High & $32(22.1)$ & $725(37.4)$ & \\
\hline Hospitals with high volume, n (\%) & & & $<0.001$ \\
\hline \multicolumn{4}{|l|}{ Physician volume } \\
\hline Low & $133(54.5)$ & 445 (26.7) & \\
\hline Medium & $64(26.2)$ & $456(27.4)$ & \\
\hline High & $47(19.3)$ & $766(46.0)$ & \\
\hline
\end{tabular}


TABLE 2

Hazard Ratio (HR) and 95\% CI of Months to Recurrence After Surgery for Prostate Cancer by Hospital Volume Categories, SEER-Medicare 1991-2002*

\begin{tabular}{|c|c|c|c|}
\hline & \multicolumn{3}{|c|}{ Hospital Volume } \\
\hline & Low HR $(95 \%$ CI) & Medium HR (95\% CI) & High HR $(95 \%$ CI $)$ \\
\hline \multicolumn{4}{|l|}{ Unadjusted } \\
\hline \multicolumn{4}{|l|}{ Race } \\
\hline White (ref) & 1.00 & 1.00 & 1.00 \\
\hline Black & $1.27(1.03-1.56)$ & $1.36(1.14-1.62)$ & $1.45(1.23-1.70)$ \\
\hline \multicolumn{4}{|l|}{ Adjusted ${ }^{\dagger}$} \\
\hline \multicolumn{4}{|l|}{ Race } \\
\hline White (ref) & 1.00 & 1.00 & 1.00 \\
\hline Black & $1.18(0.95-1.47)$ & $1.30(1.04-1.61)$ & $1.36(1.07-1.73)$ \\
\hline Age at diagnosis & $1.06(1.04-1.08)$ & $1.05(1.03-1.07)$ & $1.05(1.03-1.07)$ \\
\hline \multicolumn{4}{|l|}{ Gleason grade } \\
\hline Well differentiated (ref) & 1.00 & 1.00 & 1.00 \\
\hline Moderately differentiated & $1.00(0.77-1.31)$ & $1.47(1.05-2.07)$ & $1.66(1.21-2.26)$ \\
\hline Poorly differentiated & $1.84(1.38-2.47)$ & $2.82(1.94-4.10)$ & $2.97(2.19-4.02)$ \\
\hline \multicolumn{4}{|l|}{ Comorbidity score } \\
\hline 0 (ref) & 1.00 & 1.00 & 1.00 \\
\hline 1 & $1.18(0.99-1.39)$ & $1.31(1.10-1.55)$ & $1.39(1.16-1.67)$ \\
\hline $2+$ & $2.04(1.62-2.56)$ & $1.96(1.38-2.77)$ & $2.10(1.62-2.72)$ \\
\hline \multicolumn{4}{|l|}{ Cancer stage ${ }^{t}$} \\
\hline Stage 1 (ref) & 1.00 & 1.00 & 1.00 \\
\hline Stage 2 & $0.93(0.73-1.20)$ & $1.04(0.82-1.32)$ & $0.87(0.62-1.22)$ \\
\hline Stage 3 & $1.22(0.98-1.51)$ & $1.33(1.06-1.66)$ & $1.35(1.00-1.81)$ \\
\hline Stage 4 & $1.76(1.34-2.32)$ & $2.16(1.65-2.81)$ & $1.97(1.47-2.63)$ \\
\hline Localized/regional & $0.95(0.76-1.19)$ & $1.14(0.90-1.45)$ & $0.90(0.66-1.22)$ \\
\hline
\end{tabular}

* Hospital volume is calculated as number of prostatectomies or related surgery claims from hospital during 8 quarters before surgery, $0-63=$ Low; 64-116 = Medium; $117-371=$ High .

${ }^{\dagger}$ Also adjusted for SEER Site.

Ftages 1-4 are based on American Joint Committee on Cancer (AJCC) staging. Where AJCC staging is missing, SEER Historic Stage (which combines localized and regional stage prostate cancer) is used. 


\section{TABLE 3}

Hazard Ratio (HR) and 95\% CI of Months to Recurrence After Surgery for Prostate Cancer by Physician Volume Categories, SEER-Medicare 1991-2002*

Physician Volume

\begin{tabular}{|c|c|c|c|}
\hline & \multicolumn{3}{|c|}{ Physician Volume } \\
\hline & Low HR (95\% CI) & Medium HR (95\% CI) & High HR (95\% CI) \\
\hline \multicolumn{4}{|l|}{ Unadjusted } \\
\hline \multicolumn{4}{|l|}{ Race } \\
\hline White (ref) & 1.00 & 1.00 & 1.00 \\
\hline Black & $1.25(1.02-1.52)$ & $1.37(1.09-1.72)$ & $1.59(1.21-2.10)$ \\
\hline \multicolumn{4}{|l|}{ Adjusted ${ }^{\dagger}$} \\
\hline \multicolumn{4}{|l|}{ Race } \\
\hline White (ref) & 1.00 & 1.00 & 1.00 \\
\hline Black & $1.10(0.89-1.37)$ & $1.43(1.10-1.85)$ & $1.57(1.14-2.16)$ \\
\hline Age at diagnosis & $1.03(1.01-1.06)$ & $1.03(1.00-1.06)$ & $1.04(1.01-1.06)$ \\
\hline \multicolumn{4}{|l|}{ Gleason grade } \\
\hline Well differentiated (ref) & 1.00 & 1.00 & 1.00 \\
\hline Moderately differentiated & $0.68(0.48-0.95)$ & $1.42(0.96-2.08)$ & $1.76(1.16-2.65)$ \\
\hline Poorly differentiated & $1.41(0.99-2.00)$ & $2.95(1.97-4.41)$ & $3.11(2.00-4.85)$ \\
\hline \multicolumn{4}{|l|}{ Comorbidity score } \\
\hline 0 (ref) & 1.00 & 1.00 & 1.00 \\
\hline 1 & $1.30(1.07-1.57)$ & $1.23(1.00-1.51)$ & $1.46(1.16-1.84)$ \\
\hline $2+$ & $2.03(1.44-2.86)$ & $1.65(1.11-2.43)$ & $2.02(1.42-2.88)$ \\
\hline \multicolumn{4}{|l|}{ Cancer stage $e^{\ddagger}$} \\
\hline Stage 1 (ref) & 1.00 & 1.00 & 1.00 \\
\hline Stage 2 & $1.04(0.78-1.39)$ & $1.32(0.95-1.84)$ & $0.95(0.66-1.36)$ \\
\hline Stage 3 & $1.50(1.22-1.85)$ & $1.77(1.35-2.33)$ & $1.59(1.12-2.25)$ \\
\hline Stage 4 & $2.22(1.65-2.99)$ & $2.52(1.77-3.59)$ & $2.36(1.62-3.45)$ \\
\hline Localized/regional & $1.04(0.81-1.33)$ & $1.40(1.04-1.88)$ & $1.29(0.91-1.85)$ \\
\hline
\end{tabular}

* Physician volume is calculated as number of prostatectomies or related surgery claims for performing physician during 8 quarters before surgery, 0-9 = Low; 10-19 = Medium High; 20-77 = High.

${ }^{\dagger}$ Also adjusted for SEER Site.

F Stages 1-4 are based on American Joint Committee on Cancer (AJCC) staging. Where AJCC staging is missing, SEER Historic Stage (which combines localized and regional stage prostate cancer) is used. 